PROCEEDINGS OF THE

AMERICAN MATHEMATICAL SOCIETY

Volume 139, Number 8, August 2011, Pages 2713-2720

S 0002-9939(2011)10907-9

Article electronically published on March 22, 2011

\title{
A RESTRICTION THEOREM FOR THE H-TYPE GROUPS
}

\author{
HEPING LIU AND YINGZHAN WANG
}

(Communicated by Richard Rochberg)

\begin{abstract}
We prove that the restriction operator for the H-type groups is bounded from $L^{p}$ to $L^{p^{\prime}}$ for $p$ near to 1 when the dimension of the center is larger than one, and the range of $p$ depends on the dimension of the center. This is different from the Heisenberg group, on which the restriction operator is not bounded from $L^{p}$ to $L^{p^{\prime}}$ unless $p=1$.
\end{abstract}

\section{INTRODUCTION}

The restriction theorems for the Fourier transform play an important role in harmonic analysis as well as in the theory of partial differential equations. We refer the reader to [7] for the basic results on restriction theorems. Further developments can be found in 8 and the references therein. To generalize the restriction theorems to the Heisenberg group, D. Müller [5] investigated the restriction operator for the sublaplacian. He established the boundedness of the restriction operator with respect to the mixed $L^{p}$-norms and also gave a counterexample to show that the restriction operator is not bounded from $L^{p}$ to $L^{p^{\prime}}$, where $\frac{1}{p}+\frac{1}{p^{\prime}}=1$, unless $p=1$.

Müller's result is due to the following key fact. The center of the Heisenberg group is of dimension 1 . One can expect $L^{p} \longrightarrow L^{p^{\prime}}$ restriction theorems for some values $p>1$ on a nilpotent Lie group with the center of dimension larger than one. This has been done by S. Thangavelu [10 on products of Heisenberg groups. In [4] we prove that the restriction operator for the sublaplacian on the quaternion Heisenberg group is bounded from $L^{p}$ to $L^{p^{\prime}}$ if $1 \leq p \leq \frac{4}{3}$. In this article we will show that the restriction operator for the sublaplacian on the H-type groups is bounded from $L^{p}$ to $L^{p^{\prime}}$ for $p$ near to 1 when the dimension of the center is larger than one, and the range of $p$ depends on the dimension of the center.

First we state some facts of H-type groups. We refer the reader to [1, 2] and 13 for the basic references of H-type groups. Let $\mathfrak{g}$ be a two step nilpotent Lie algebra endowed with an inner product $\langle$,$\rangle . Its center is denoted by \mathfrak{Z}$. $\mathfrak{g}$ is called an H-type algebra if $\left[\mathfrak{Z}^{\perp}, \mathfrak{Z}^{\perp}\right]=\mathfrak{Z}$ and for every $t \in \mathfrak{Z}$, the map $J_{t}: \mathfrak{Z}^{\perp} \rightarrow \mathfrak{Z}^{\perp}$ defined by

$$
\left\langle J_{t} u, w\right\rangle=\langle t,[u, w]\rangle, \quad \text { for any } u, w \in \mathfrak{Z}^{\perp},
$$

Received by the editors May 12, 2010.

2010 Mathematics Subject Classification. Primary 42B10, 43A65.

Key words and phrases. H-type group, restriction operator, special Hermite expansion.

The authors were supported by the National Natural Science Foundation of China under Grants \#10871003 and \#10990012, and the Specialized Research Fund for the Doctoral Program of Higher Education of China under Grant \#2007001040.

(C)2011 American Mathematical Society Reverts to public domain 28 years from publication 2713 
is an orthogonal map whenever $|t|=1$. The corresponding connected and simply connected Lie group $G$ is called an H-type group. For a given $0 \neq a \in \mathfrak{Z}^{*}$, the dual of $\mathfrak{Z}$, we can define a skew-symmetric linear mapping $B(a)$ on $\mathfrak{Z}^{\perp}$ by

$$
\langle B(a) u, w\rangle=a([u, w]), \quad \text { for any } u, w \in \mathfrak{Z}^{\perp} .
$$

$B(a)$ is non-degenerate and we have

$$
\operatorname{dim} \mathfrak{Z}^{\perp}=2 n .
$$

Thus we can choose an orthonormal basis

$$
\left\{E_{1}(a), E_{2}(a), \cdots, E_{n}(a), \bar{E}_{1}(a), \bar{E}_{2}(a), \cdots, \bar{E}_{n}(a)\right\}
$$

of $\mathfrak{Z}^{\perp}$ such that

$$
B(a) E_{i}(a)=-|a| \bar{E}_{i}(a), B(a) \bar{E}_{i}(a)=|a| E_{i}(a) .
$$

We also choose an orthonormal basis $\left\{\varepsilon_{1}, \varepsilon_{2}, \cdots, \varepsilon_{m}\right\}$ of $\mathfrak{Z}$ such that $a\left(\varepsilon_{1}\right)=$ $|a|, a\left(\varepsilon_{j}\right)=0, j=2, \cdots, m$. Throughout this paper we will assume that $m>1$. Then the elements of $\mathfrak{g}$ can be written as $(z, t)=(x, y, t)=\sum_{i=1}^{n}\left(x_{i} E_{i}+y_{i} \bar{E}_{i}\right)+$ $\sum_{j=1}^{m} t_{j} \varepsilon_{j}$. We identify $G$ with its Lie algebra $\mathfrak{g}$ by the exponential map. The group structure is given by

$$
(z, t)\left(z^{\prime}, t^{\prime}\right)=\left(z+z^{\prime}, t+t^{\prime}+\frac{1}{2}\left[z, z^{\prime}\right]\right)
$$

where $\left[z, z^{\prime}\right]_{j}=\left\langle z, U^{j} z^{\prime}\right\rangle$, and $U^{j}$ satisfies the following conditions:

(i) $U^{j}$ is a $2 n \times 2 n$ skew-symmetric and orthogonal matrix, $j=1,2, \cdots, m$,

(ii) $U^{i} U^{j}+U^{j} U^{i}=0, \quad i, j=1,2, \cdots, m$.

In particular, $\left\langle z, U^{1} z^{\prime}\right\rangle=\sum_{j=1}^{n}\left(x_{j}^{\prime} y_{j}-x_{j} y_{j}^{\prime}\right)$. We remark that all the above expressions depend on a given $0 \neq a \in \mathfrak{Z}^{*}$, but we omit it. We will keep this simplification.

The left invariant vector fields which agree respectively with $\frac{\partial}{\partial x_{j}}, \frac{\partial}{\partial y_{j}}, j=1,2$, $\cdots, n$, at the origin are given by

$$
\begin{aligned}
X_{j} & =\frac{\partial}{\partial x_{j}}+\frac{1}{2} \sum_{k=1}^{m}\left(\sum_{l=1}^{2 n} z_{l} U_{l, j}^{k}\right) \frac{\partial}{\partial t_{k}} \\
Y_{j} & =\frac{\partial}{\partial y_{j}}+\frac{1}{2} \sum_{k=1}^{m}\left(\sum_{l=1}^{2 n} z_{l} U_{l, j+n}^{k}\right) \frac{\partial}{\partial t_{k}}
\end{aligned}
$$

where $z_{l}=x_{l}, z_{l+n}=y_{l}, l=1,2, \cdots, n$. Then the sublaplacian is defined by

$$
\mathcal{L}=-\sum_{j=1}^{n}\left(X_{j}^{2}+Y_{j}^{2}\right)=-\Delta_{z}-\frac{1}{4}|z|^{2} \Delta_{t}-\sum_{k=1}^{m}\left\langle z, U^{k} \nabla_{z}\right\rangle \frac{\partial}{\partial t_{k}}
$$

where

$$
\Delta_{z}=\sum_{j=1}^{2 n} \frac{\partial^{2}}{\partial z_{j}^{2}}, \quad \Delta_{t}=\sum_{j=1}^{m} \frac{\partial^{2}}{\partial t_{j}^{2}}, \quad \nabla_{z}=\left(\frac{\partial}{\partial z_{1}}, \frac{\partial}{\partial z_{2}}, \cdots, \frac{\partial}{\partial z_{2 n}}\right)^{T} .
$$

It is well known that the sublaplacian $\mathcal{L}$ is positive and essentially self-adjoint. Let

$$
\mathcal{L}=\int_{0}^{\infty} \lambda d E(\lambda)
$$


be the spectral decomposition of $\mathcal{L}$. Then operator

$$
P_{\lambda} f=\lim _{\varepsilon \rightarrow 0} \frac{1}{\varepsilon} \int_{\lambda}^{\lambda+\varepsilon} d E(\lambda) f
$$

is well defined for $f \in \mathscr{S}(G)$. Thus

$$
f=\int_{0}^{\infty} P_{\lambda} f d \lambda
$$

where $P_{\lambda} f$ is an eigenfunction of $\mathcal{L}$ with the eigenvalue $\lambda . P_{\lambda}$ is called the restriction operator for the sublaplacian $\mathcal{L}$. Our main result is the following theorem.

Theorem 1. Let $G$ be an H-type group with the underlining manifold $\mathbb{R}^{2 n+m}$, where $m>1$ is the dimension of the center. Then for $1 \leq p \leq \frac{2 m+2}{m+3}$,

$$
\left\|P_{\lambda} f\right\|_{p^{\prime}} \leq C \lambda^{2(n+m)\left(\frac{1}{p}-\frac{1}{2}\right)-1}\|f\|_{p}
$$

This article is organized as follows. In the next section we give an explicit expression for the restriction operator. Theorem 1 will be proved in the last section.

\section{RESTRICTION OPERATOR}

Let us recall some results about the special Hermite expansion. We refer to [11, 12 for details. The Hermite polynomials $H_{k}(x)$ are defined on $\mathbb{R}$ by the formula

Set

$$
H_{k}(x)=(-1)^{k} \frac{d^{k}}{d x^{k}}\left(e^{-x^{2}}\right) e^{x^{2}}, \quad k=0,1,2, \cdots
$$

$$
h_{k}(x)=\left(2^{k} k ! \sqrt{\pi}\right)^{-\frac{1}{2}} H_{k}(x) e^{-\frac{1}{2} x^{2}}, \quad k=0,1,2, \cdots .
$$

Let $\alpha$ be a multiindex and $x \in \mathbb{R}^{n}$. We define Hermite functions $\Phi_{\alpha}$ by

$$
\Phi_{\alpha}(x)=\prod_{j=1}^{n} h_{\alpha_{j}}\left(x_{j}\right)
$$

For each pair of multiindices $\alpha, \beta$, and $z=x+i y \in \mathbb{C}^{n}$, the special Hermite function $\Phi_{\alpha \beta}$ is defined in terms of the Fourier-Wigner transform of $\Phi_{\alpha}$ and $\Phi_{\beta}$, which is essentially the matrix coefficient of the Schrödinger representation denoted by $\pi$, i.e.,

$$
\Phi_{\alpha \beta}(z)=(2 \pi)^{-\frac{n}{2}} \int_{\mathbb{R}^{n}} e^{i x \cdot \xi} \Phi_{\alpha}\left(\xi+\frac{y}{2}\right) \Phi_{\beta}\left(\xi-\frac{y}{2}\right) d \xi .
$$

The special Hermite functions form a complete orthonormal system for $L^{2}\left(\mathbb{C}^{n}\right)$. Thus for $f \in L^{2}\left(\mathbb{C}^{n}\right)$, we have the special Hermite expansion

$$
f=\sum_{\alpha \in \mathbb{N}^{n}} \sum_{\beta \in \mathbb{N}^{n}}\left\langle f, \Phi_{\alpha \beta}\right\rangle \Phi_{\alpha \beta}
$$

The special Hermite expansion can be put in a compact form. For $f, g \in L^{1}\left(\mathbb{C}^{n}\right)$, we define the twisted convolution $f \times g$ by

$$
f \times g(z)=\int_{\mathbb{C}^{n}} f(z-w) g(w) e^{\frac{i}{2} \operatorname{Im} z \cdot \bar{w}} d w .
$$

Then

$$
\sum_{\beta \in \mathbb{N}^{n}}\left\langle f, \Phi_{\beta \alpha}\right\rangle \Phi_{\beta \alpha}(z)=(2 \pi)^{-\frac{n}{2}} f \times \Phi_{\alpha \alpha}(z) .
$$


Let $L_{k}^{\nu}(x)$ be the Laguerre polynomial of type $\nu$ and degree $k$ defined by

$$
L_{k}^{\nu}(x)=\frac{1}{k !} \frac{d^{k}}{d x^{k}}\left(e^{-x} x^{k+\nu}\right) e^{x} x^{-\nu}, \quad k=0,1,2, \cdots, \nu>-1 .
$$

Set

$$
\varphi_{k}(z)=L_{k}^{n-1}\left(\frac{1}{2}|z|^{2}\right) e^{-\frac{1}{4}|z|^{2}}, \quad k=0,1,2, \cdots
$$

Then

$$
\varphi_{k}(z)=(2 \pi)^{\frac{n}{2}} \sum_{|\alpha|=k} \Phi_{\alpha \alpha}(z) .
$$

Thus we can rewrite the special Hermite expansion as

$$
f=(2 \pi)^{-n} \sum_{k=0}^{\infty} f \times \varphi_{k} .
$$

Let $L$ be the special Hermite operator defined by

$$
L=-\Delta_{z}+\frac{|z|^{2}}{4}-i \sum_{j=1}^{n}\left(x_{j} \frac{\partial}{\partial y_{j}}-y_{j} \frac{\partial}{\partial x_{j}}\right),
$$

where we have identified $z=x+i y \in \mathbb{C}^{n}$ with $z=(x, y) \in \mathbb{R}^{2 n}$. For $1 \leq p<\frac{6 n+2}{3 n+4}$, we have

$$
\left\|f \times \varphi_{k}\right\|_{2} \leq C(k+1)^{n\left(\frac{1}{p}-\frac{1}{2}\right)-\frac{1}{2}}\|f\|_{p}
$$

(see [9] or [12, p. 72]).

We need to consider the scaled special Hermite expansion. Let $\lambda>0$. The scaled special Hermite operator is defined by

$$
L_{\lambda}=-\Delta_{z}+\frac{\lambda^{2}|z|^{2}}{4}-i \lambda \sum_{j=1}^{n}\left(x_{j} \frac{\partial}{\partial y_{j}}-y_{j} \frac{\partial}{\partial x_{j}}\right) .
$$

Set $\varphi_{k}^{\lambda}(z)=\varphi_{k}(\sqrt{\lambda} z)$ and define the $\lambda$-twisted convolution by

$$
f \times_{\lambda} g(z)=\int_{\mathbb{C}^{n}} f(z-w) g(w) e^{\frac{i \lambda}{2} \operatorname{Im} z \cdot \bar{w}} d w .
$$

By a dilation argument, for $f \in \mathscr{S}\left(\mathbb{C}^{n}\right)$, we have

$$
f(z)=\left(\frac{\lambda}{2 \pi}\right)^{n} \sum_{k=0}^{\infty} f \times_{\lambda} \varphi_{k}^{\lambda}(z)
$$

and

$$
\|f\|_{2}^{2}=\left(\frac{\lambda}{2 \pi}\right)^{2 n} \sum_{k=0}^{\infty}\left\|f \times_{\lambda} \varphi_{k}^{\lambda}\right\|_{2}^{2} .
$$

Moreover, $f \times_{\lambda} \varphi_{k}^{\lambda}$ is an eigenfunction of $L_{\lambda}$ with the eigenvalue $(2 k+n) \lambda$, and

$$
\left\|f \times_{\lambda} \varphi_{k}^{\lambda}\right\|_{2} \leq C(k+1)^{n\left(\frac{1}{p}-\frac{1}{2}\right)-\frac{1}{2}} \lambda^{n\left(\frac{1}{p}-\frac{3}{2}\right)}\|f\|_{p} \text { for } 1 \leq p<\frac{6 n+2}{3 n+4} .
$$

Now we turn to the expression for the restriction operator. We may identify $\mathfrak{Z}^{*}$ with $\mathfrak{Z}$. Thus we will write $\langle a, t\rangle$ instead of $a(t)$ for $a \in \mathfrak{Z}^{*}$ and $t \in \mathfrak{Z}$.

Lemma 1. Let $0 \neq a \in \mathfrak{Z}^{*}$. If $f(z, t)=e^{-i\langle a, t\rangle} \varphi(z)$, then

$$
\mathcal{L} f(z, t)=e^{-i\langle a, t\rangle} L_{|a|} \varphi(z) .
$$


Proof. Because $\langle a, t\rangle=|a| t_{1}$ and

$$
\left\langle z, U^{1} \nabla_{z}\right\rangle=\sum_{j=1}^{n}\left(y_{j} \frac{\partial}{\partial x_{j}}-x_{j} \frac{\partial}{\partial y_{j}}\right),
$$

Lemma 1 is easily deduced from the expression (1.1).

Set $e_{k}^{a}(z, t)=e^{-i\langle a, t\rangle} \varphi_{k}^{|a|}(z)$. For $f \in \mathscr{S}(G)$, let

$$
f^{a}(z)=\int_{\mathbb{R}^{m}} f(z, t) e^{i\langle a, t\rangle} d t
$$

be the Fourier transform of $f$ with respect to the central variable $t$. It is easy to get

$$
f * e_{k}^{a}(z, t)=e^{-i\langle a, t\rangle}\left(f^{a} \times_{|a|} \varphi_{k}^{|a|}\right)(z) .
$$

It follows from Lemma 1 that $f * e_{k}^{a}$ is an eigenfunction of $\mathcal{L}$ with the eigenvalue $(2 k+n)|a|$.

Let $\tilde{e}_{k}^{a}=e_{k}^{\frac{a}{2 k+n}}$. Using the inversion formula for the Fourier transform together with (2.1) and (2.4), we get

$$
\begin{aligned}
f(z, t) & =\frac{1}{(2 \pi)^{m}} \int_{\mathbb{R}^{m}} f^{a}(z) e^{-i\langle a, t\rangle} d a \\
& =\frac{1}{(2 \pi)^{m}} \int_{\mathbb{R}^{m}}\left(\frac{|a|^{n}}{(2 \pi)^{n}} \sum_{k=0}^{\infty} f^{a} \times_{|a|} \varphi_{k}^{|a|}(z)\right) e^{-i\langle a, t\rangle} d a \\
& =\frac{1}{(2 \pi)^{n+m}} \sum_{k=0}^{\infty} \int_{\mathbb{R}^{m}} f * e_{k}^{a}(z, t)|a|^{n} d a \\
& =\frac{1}{(2 \pi)^{n+m}} \sum_{k=0}^{\infty} \frac{1}{(2 k+n)^{n+m}} \int_{\mathbb{R}^{m}} f * \tilde{e}_{k}^{a}(z, t)|a|^{n} d a \\
& =\int_{0}^{\infty}\left(\sum_{k=0}^{\infty} \frac{\lambda^{n+m-1}}{(2 \pi(2 k+n))^{n+m}} \int_{S^{m-1}} f * \tilde{e}_{k}^{\lambda \tilde{a}}(z, t) d \sigma(\tilde{a})\right) d \lambda
\end{aligned}
$$

where $\tilde{a}=\frac{a}{|a|}$ and $d \sigma(\tilde{a})$ is the induced Euclidean measure on the unit sphere $S^{m-1}$. Thus

$$
P_{\lambda} f(z, t)=\sum_{k=0}^{\infty} \frac{\lambda^{n+m-1}}{(2 \pi(2 k+n))^{n+m}} \int_{S^{m-1}} f * \tilde{e}_{k}^{\lambda \tilde{a}}(z, t) d \sigma(\tilde{a}) .
$$

We also have, by the Plancherel formula and (2.2),

$$
\begin{aligned}
\|f\|_{L^{2}(G)}^{2} & =(2 \pi)^{-m} \int_{\mathbb{R}^{m}} \int_{\mathbb{R}^{2 n}}\left|f^{a}(z)\right|^{2} d z d a \\
& =\sum_{k=0}^{\infty}(2 \pi)^{-m} \int_{\mathbb{R}^{m}} \int_{\mathbb{R}^{2 n}} \frac{|a|^{2 n}}{(2 \pi)^{2 n}}\left|f^{a} \times_{|a|} \varphi_{k}^{|a|}(z)\right|^{2} d z d a \\
& =\sum_{k=0}^{\infty} \frac{1}{(2 \pi)^{2 n+m}} \int_{\mathbb{R}^{m}}|a|^{2 n} \int_{\mathbb{R}^{2 n}}\left|f * e_{k}^{a}(z, 0)\right|^{2} d z d a \\
& =\sum_{k=0}^{\infty} \frac{1}{(2 \pi(2 k+n))^{2 n+m}} \int_{\mathbb{R}^{m}}|a|^{2 n} \int_{\mathbb{R}^{2 n}}\left|f * \tilde{e}_{k}^{a}(z, 0)\right|^{2} d z d a .
\end{aligned}
$$


Set

$$
Q(a, f)^{2}=\sum_{k=0}^{\infty} \frac{|a|^{2 n}}{(2 \pi(2 k+n))^{2 n+m}} \int_{\mathbb{R}^{2 n}}\left|f * \tilde{e}_{k}^{a}([z, 0])\right|^{2} d z
$$

Then

$$
\|f\|_{2}^{2}=\int_{\mathbb{R}^{m}} Q(a, f)^{2} d a
$$

\section{Restriction theorem}

In this section we prove Theorem 1 .

Lemma 2. Letting $1 \leq p \leq \frac{2 m+2}{m+3}$, we have

$$
\left(\int_{S^{m-1}} Q(\lambda \tilde{a}, f)^{q} d \sigma(\tilde{a})\right)^{\frac{1}{q}} \leq C \lambda^{\frac{n+m}{p}-\frac{n}{2}-m}\|f\|_{p}, \quad q=\frac{m-1}{m+1} p^{\prime} .
$$

Because $\frac{m-1}{m+1} p^{\prime} \geq 2$, it follows that

$$
\left(\int_{S^{m-1}} Q(\lambda \tilde{a}, f)^{2} d \sigma(\tilde{a})\right)^{\frac{1}{2}} \leq C \lambda^{\frac{n+m}{p}-\frac{n}{2}-m}\|f\|_{p} .
$$

Proof. Note that

$$
Q(\lambda \tilde{a}, f)^{2}=\sum_{k=0}^{\infty} \frac{\lambda^{2 n}}{(2 \pi(2 k+n))^{2 n+m}} \int_{\mathbb{R}^{2 n}}\left|f^{\frac{\lambda \tilde{a}}{2 k+n}} \times \frac{\lambda}{2 k+n} \varphi_{k}^{\frac{\lambda}{2 k+n}}(z)\right|^{2} d z .
$$

It is well known that H-type algebras are closely related to Clifford modules (cf. [6]). H-type algebras can be classified by the standard theory of Clifford algebras. Specifically, the relation of the dimensions of the center to its orthogonal complement space is clear. In any case, we have $m<2 n$ (cf. [3]). Thus $\frac{2 m+2}{m+3}<\frac{6 n+2}{3 n+4}$. By (2.3),

$$
\left\|f^{\frac{\lambda \tilde{a}}{2 k+n}} \times \frac{\lambda}{2 k+n} \varphi_{k}^{\frac{\lambda}{2 k+n}}\right\|_{2}^{2} \leq C \lambda^{-3 n+\frac{2 n}{p}}(k+1)^{2 n-1}\left\|f^{\frac{\lambda \tilde{a}}{2 k+n}}\right\|_{p}^{2} .
$$

Because $q=\frac{m-1}{m+1} p^{\prime} \geq 2>p$, by (3.3) and the Minkowski inequality, we get

$$
\begin{aligned}
& \left(\int_{S^{m-1}} Q(\lambda \tilde{a}, f)^{q} d \sigma(\tilde{a})\right)^{\frac{1}{q}} \\
\leq & C \sum_{k=0}^{\infty} \frac{\lambda^{n}}{(k+1)^{\frac{2 n+m}{2}}}\left(\int_{S^{m-1}}\left(\int_{\mathbb{R}^{2 n}}\left|f^{\frac{\lambda \tilde{a}}{2 k+n}} \times \frac{\lambda}{2 k+n} \varphi_{k}^{\frac{\lambda}{2 k+n}}(z)\right|^{2} d z\right)^{\frac{q}{2}} d \sigma(\tilde{a})\right)^{\frac{1}{q}} \\
\leq & C \sum_{k=0}^{\infty} \lambda^{n\left(\frac{1}{p}-\frac{1}{2}\right)}(k+1)^{-\frac{m+1}{2}}\left(\int_{S^{m-1}}\left(\int_{\mathbb{R}^{2 n}}\left|f^{\frac{\lambda \tilde{a}}{2 k+n}}(z)\right|^{p} d z\right)^{\frac{q}{p}} d \sigma(\tilde{a})\right)^{\frac{1}{q}} \\
\leq & C \sum_{k=0}^{\infty} \lambda^{n\left(\frac{1}{p}-\frac{1}{2}\right)}(k+1)^{-\frac{m+1}{2}}\left(\int_{\mathbb{R}^{2 n}}\left(\int_{S^{m-1}}\left|f^{\frac{\lambda \tilde{a}}{2 k+n}}(z)\right|^{q} d \sigma(\tilde{a})\right)^{\frac{p}{q}} d z\right)^{\frac{1}{p}} \\
\leq & C \sum_{k=0}^{\infty} \lambda^{n\left(\frac{1}{p}-\frac{1}{2}\right)-m}(k+1)^{\frac{m-1}{2}}\left(\int_{\mathbb{R}^{2 n}}\left(\int_{S^{m-1}}\left|\tilde{f}_{k}^{\tilde{a}}(z)\right|^{q} d \sigma(\tilde{a})\right)^{\frac{p}{q}} d z\right)^{\frac{1}{p}}
\end{aligned}
$$


where $\tilde{f}_{k}(z, t)=f\left(z, \frac{2 k+n}{\lambda} t\right)$. It follows from the restriction estimate of the Fourier transform on $S^{m-1}$ (see [7, p. 386]) that

$$
\left(\int_{S^{m-1}}\left|\tilde{f}_{k}^{\tilde{a}}(z)\right|^{q} d \sigma(\tilde{a})\right)^{\frac{1}{q}} \leq C\left(\int_{\mathbb{R}^{m}}\left|\tilde{f}_{k}(z, t)\right|^{p} d t\right)^{\frac{1}{p}} .
$$

Therefore,

$$
\begin{aligned}
\left(\int_{S^{m-1}}\right. & \left.Q(\lambda \tilde{a}, f)^{q} d \sigma(\tilde{a})\right)^{\frac{1}{q}} \\
\leq & C \sum_{k=0}^{\infty} \lambda^{n\left(\frac{1}{p}-\frac{1}{2}\right)-m}(k+1)^{\frac{m-1}{2}}\left(\int_{\mathbb{R}^{2 n}} \int_{\mathbb{R}^{m}}\left|\tilde{f}_{k}(z, t)\right|^{p} d t d z\right)^{\frac{1}{p}} \\
\leq & C \sum_{k=0}^{\infty} \lambda^{\frac{n}{p}-\frac{n}{2}-m+\frac{m}{p}} k^{\frac{m-1}{2}-\frac{m}{p}}\|f\|_{p} \\
\leq & C \lambda^{\frac{n}{p}-\frac{n}{2}-m+\frac{m}{p}}\|f\|_{p} .
\end{aligned}
$$

Now we give the proof of Theorem 1.

Proof of Theorem 1. Because the special Hermite expansion is an orthogonal expansion, we have

$$
\begin{aligned}
\left\langle P_{\lambda} f, g\right\rangle & =\int_{G} P_{\lambda} f(z, t) \overline{g(z, t)} d z d t \\
& =\int_{G}\left(\sum_{k=0}^{\infty} \frac{\lambda^{n+m-1}}{(2 \pi(2 k+n))^{n+m}} \int_{S^{m-1}} f * \tilde{e}_{k}^{\lambda \tilde{a}}(z, t) d \sigma(\tilde{a})\right) \overline{g(z, t)} d z d t \\
& =\int_{S^{m-1}} \sum_{k=0}^{\infty} \int_{\mathbb{R}^{2 n}} \frac{\lambda^{n+m-1}}{(2 \pi(2 k+n))^{n+m}} f^{\frac{\lambda \tilde{a}}{2 k+n}} \times \frac{\lambda}{2 k+n} \varphi_{k}^{\frac{\lambda}{2 k+n}}(z) \\
& =\int_{S^{m-1}} \sum_{k=0}^{\infty} \int_{\mathbb{R}^{2 n}} \frac{\lambda^{2 n+m-1}}{g^{\frac{\lambda \tilde{a}}{2 k+n}}(z)} d z d \sigma(\tilde{a}) \\
& \leq C \lambda^{m-1}\|Q(\lambda \tilde{a}, f)\|_{L^{2}\left(S^{m-1}\right)}\|Q(\lambda \tilde{a}, g)\|_{L^{2}\left(S^{m-1}\right)} \cdot \frac{\frac{\lambda \tilde{a}}{2 k+n}}{g_{\frac{\lambda}{2 k+n}}} \varphi_{k}^{\frac{\lambda}{2 k+n}}(z)
\end{aligned}
$$

By (3.2), when $1 \leq p \leq \frac{2 m+2}{m+3}$, we have

$$
\left\langle P_{\lambda} f, g\right\rangle \leq C \lambda^{2(n+m)\left(\frac{1}{p}-\frac{1}{2}\right)-1}\|f\|_{p}\|g\|_{p} .
$$

So

$$
\left\|P_{\lambda} f\right\|_{p^{\prime}}=\sup _{\|g\|_{p}=1}\left|\left\langle P_{\lambda} f, g\right\rangle\right| \leq C \lambda^{2(n+m)\left(\frac{1}{p}-\frac{1}{2}\right)-1}\|f\|_{p} .
$$

Theorem 1 is proved. 


\section{REFERENCES}

1. M. Cowling, A. H. Dooley, A. Korányi and F. Ricci, H-type groups and Iwasawa decompositions, Adv. Math. 87 (1991), no. 1, 1-41. MR1102963 (92e:22017)

2. A. Kaplan, Fundamental solutions for a class of hypoelliptic PDE generated by composition of quadratic forms, Trans. Amer. Math. Soc. 258 (1980), no. 1, 147-153. MR.554324 (81c:58059)

3. A. Kaplan and F. Ricci, Harmonic analysis on groups of Heisenberg type, Harmonic analysis, Lecture Notes in Math., 992, Springer, Berlin, 1983, 416-435. MR729367 (85h:22017)

4. H. Liu and Y. Wang, A restriction theorem for the quaternion Heisenberg group, Appl. Math. J. Chinese Univ. Series B., to appear.

5. D. Müller, A restriction theorem for the Heisenberg group, Ann. of Math. (2) 131 (1990), no. 3, 567-587. MR.1053491 (91k:22021)

6. H. M. Reimann, H-type groups and Clifford modules, Adv. Appl. Clifford Algebras 11 (2001), no. S2, 277-287. MR2075359 (2005j:15033)

7. E. M. Stein, Harmonic analysis: real-variable methods, orthogonality, and oscillatory integrals, Princeton Univ. Press, Princeton, N.J., 1993. MR.1232192 (95c:42002)

8. T. Tao, Some recent progress on the restriction conjecture, Fourier analysis and convexity, 217-243, Appl. Numer. Harmon. Anal., Birkhäuser Boston, 2004. MR2087245 (2005i:42015)

9. S. Thangavelu, Some restriction theorems for the Heisenberg group, Studia Math. 99 (1991), no. 1, 11-21. MR.1120736 (93e:43009)

10. S. Thangavelu, Restriction theorems for the Heisenberg group, J. Reine Angew. Math. 414 (1991), 51-65. MR.1092623 (92e:22020)

11. S. Thangavelu, Lectures on Hermite and Laguerre expansions, Princeton Univ. Press, 1993. MR.1215939 (94i:42001)

12. S. Thangavelu, Harmonic Analysis on the Heisenberg Group, Progress in Math., 159, Birkhäuser, 1998. MR1633042(99h:43001)

13. Q. Yang and F. Zhu, The heat kernel on H-type groups, Proc. Amer. Math. Soc. 136 (2008), no. 4, 1457-1464. MR2367120(2009h:35054)

Lmam, School of Mathematical Sciences, Peking University, Beijing 100871, PeoPLE'S REPUBLIC OF CHINA

E-mail address: hpliu@pku.edu.cn

College of Mathematics and Information Sciences, Guangzhou University, GuangZhou 510006, People's Republic of China

E-mail address: wyzde@tom.com 